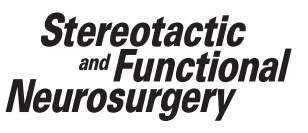

Stereotactic

Neurosurgery
Stereotact Funct Neurosurg 2013;91:148-152

DOI: $\underline{10.1159 / 000345268}$
Received: November 21, 2011

Accepted after revision: October 15, 2012

Published online: February 27, 2013

\title{
Effect of Data Normalization on the Creation of Neuro-Probabilistic Atlases
}

\author{
Pierre-François D'Haese ${ }^{\mathrm{a}}$ Srivatsan Pallavaram ${ }^{\mathrm{a}}$ Chris Kao $^{\mathrm{b}} \quad$ Joseph S. Neimat $^{\mathrm{b}}$ \\ Peter E. Konrad ${ }^{b}$ Benoit M. Dawant ${ }^{a}$ \\ ${ }^{a}$ Department of Electrical Engineering and Computer Science, Vanderbilt University, and \\ ${ }^{b}$ Department of Neurosurgery, Vanderbilt University Medical Center, Nashville, Tenn., USA
}

\author{
Key Words \\ Atlas creation - Normalization - Deep brain stimulation - \\ Brain mapping
}

\begin{abstract}
In the past 15 years, rapid improvements in imaging technology and methodology have had a tremendous impact on how we study the human brain. During deep brain stimulation surgeries, detailed anatomical images can be combined with physiological data obtained by microelectrode recordings and microstimulations to address questions relating to the location of specific motor or sensorial functions. The main advantage of techniques such as microelectrode recordings and microstimulations over brain imaging is their ability to localize patient physiological activity with a high degree of spatial resolution. Aggregating data acquired from large populations permits to build what are commonly referred to as statistical atlases. Data points from statistical atlases can be combined to produce probabilistic maps. A crucial step in this process is the intersubject spatial normalization that is required to relate a position in one subject's brain to a position in another subject's brain. In this paper, we study the impact of spatial normalization techniques on building statistical atlases. We find that the Talairach or anterior-posterior commissure coordinate system commonly used in the medical literature produces atlases that are more dispersed than those obtained with normalization methods
\end{abstract}

that rely on nonlinear volumetric image registration. We also find that the maps produced using nonlinear techniques correlate with their expected anatomic positions.

Copyright $\odot 2013$ S. Karger AG, Basel

\section{Introduction}

Brain mapping is a set of neuroscience techniques predicated on the mapping of biological quantities or properties onto spatial representations of the brain. In deep brain stimulation (DBS) neurosurgeries, brain mapping is based on intraoperative electrophysiological recordings made to identify optimal locations for the implantation of electrodes. Since the pioneering days of DBS, data have been collected, spatially normalized, analyzed, and interpreted to study the location of motor and sensorial brain functions. One approach is to build probabilistic maps that associate with every voxel in the brain image, an index that represents the likelihood of observing a particular response to the experiment of interest [1-4]. When populating these maps, the estimation of the location of functional areas in the brain is affected by two main sources of errors: (1) the error in the actual anatomical location of the measurement in a particular subject, and (2) the error made when normalizing data sets across subjects.

The first error is dependent on a number of factors. In DBS procedures, recording and stimulating electrodes

\section{KARGER}

E-Mail karger@karger.com

www.karger.com/sfn
(C) 2013 S. Karger AG, Basel

$1011-6125 / 13 / 0913-0148 \$ 38.00 / 0$
Pierre-François D'Haese, $\mathrm{PhD}$

Department of Electrical Engineering and Computer Science

Box 1679, Station B, Vanderbilt University

Nashville, TN 37235 (USA)

E-Mail pf.dhaese@vanderbilt.edu 
are most often tracked with respect to a preoperative scan by using a stereotactic frame affixed to the patient's skull. We have reported that localization errors for the stereotactic frame used at our institution are negligible and comparable to commonly used stereotactic frames $[5,6]$. Another factor is that the brain is subject to several forces that can make it shift during the procedure $[7,8]$. In 2009 , we reported that brain shift occurs in DBS surgeries with evidence of tissue displacement around the target of as much as $4.06 \mathrm{~mm}$ and studied its impact on the creation of probabilistic maps [9]. Techniques to track this shift are being developed but have not yet been proven to be accurate enough for DBS surgeries that require submillimetric precision [10]. Our surgical team minimizes this error by making small burr holes, and using gel foam and fiber sealants.

To address the second source of error, in this study, we quantify the effect of spatial normalization methods. Spatial normalization is a key element related to statistical analysis as it affects the spatial resolution of a study that involves a direct comparison across subjects. One purpose of spatial normalization is to bring homologous areas into their closest possible alignment, but this can be difficult to assess because of the lack of a gold standard against which to compare. The stereotactic or Talairach coordinate system [11] has found the most widespread acceptance in the clinical community to normalize DBSrelated observations for localization and communication of 3-dimensional positions in the brain. It uses the anterior and posterior commissures as internal landmarks to define a right-handed coordinate system. Their midpoint typically defines the origin even though either the anterior or posterior commissure is sometimes used instead. A point in the 3-dimensional space is then defined as being anterior-posterior, medial-lateral or dorsal-ventral to this origin. We have shown in the past that there is substantial intersurgeon variability in the selection of these commissures which define the Talairach reference system and that nonlinear image registration or normalization can be used to reduce this variability $[12,13]$. Nonlinear or nonrigid normalization methods use a mathematical measure of overall image mismatch and a minimization algorithm that iteratively changes the transformations in order to find the best set of parameters to match the image to the reference volume. Usually, they begin by optimizing linear parameters: translations, rotations, scaling, and often shear as well. They then proceed to find the best set of nonlinear (warping) parameters to further match the morphological details of the reference brain. Pluim et al. [14] provide a detailed survey of mutual-information-based methods [15-17] for medical image registration. Chakravarty et al. [18] survey various atlas warping techniques used for DBS and suggest that template-based atlas-to-patient warping techniques such as the methods above that use MRI for atlas creation work best for customizing the atlas to patient data.

In this study, we compare the effect of using the Talairach coordinates versus those obtained by nonlinear normalization on the creation of statistical atlases to investigate whether the normalization scheme has a substantial effect on their quality.

\section{Materials and Methods}

For the purpose of this study, we intraoperatively collected 1,099 stimulation response data points from 108 DBS ventral intermediate nucleus (Vim) implantations in 100 patients who underwent Vim DBS surgery between February 2004 and June 2010. Of these, only those that recorded at least $50 \%$ efficacy in tremor reduction (measured subjectively by a neurologist during surgery) were chosen to represent successful tremor relief. Since our intraoperative symptom assessments are performed subjectively by one of our several neurologists, it was decided that $50 \%$ symptom reduction was a good threshold because it was agreed upon as the minimum threshold required to consider symptom improvement to be acceptable in our cases and also because it was sufficient to reduce the interneurologist variability in subjective assessment. This resulted in 620 data points from 86 implantations. The surgeries were performed at the Vanderbilt University Medical Center by two neurosurgeons (co-authors P.E.K. and J.S.N.).

The following types of spatial normalization schemes were compared: (1) the use of the stereotactic or Talairach coordinates of each data point, and (2) a 3-dimensional volumetric nonlinear image registration algorithm called the Adaptive Bases Algorithm (ABA) [19] that automatically registers a subject's brain MRI to the MRI of the reference volume called the atlas. ABA combines an affine registration (9 degrees of freedom: translation, rotation and anisotropic scaling) followed by a nonrigid registration. The nonrigid registration algorithm computes a deformation field that is modeled as a linear combination of radial basis functions with finite support. This results in a transformation with several thousands of degrees of freedom. Two transformations (one from the atlas to the subject and the other from the subject to the atlas) that are constrained to be inverses of each other are computed simultaneously. ABA reduces the computational complexity and improves the convergence properties of related spline-based approaches [20-23] by identifying regions of misregistration and adapting the compliance of the transformation locally. The algorithm arrives at the final deformation iteratively across scales and resolutions, starting with large-scale transformations computed on downsampled images and progressing to local transformation computing a maximum resolution. Once normalized, each data point is used to create functional atlases of stimulation response data.

We demonstrate the effect of the spatial normalization by showing the differences in the localization and shape of probabi- 

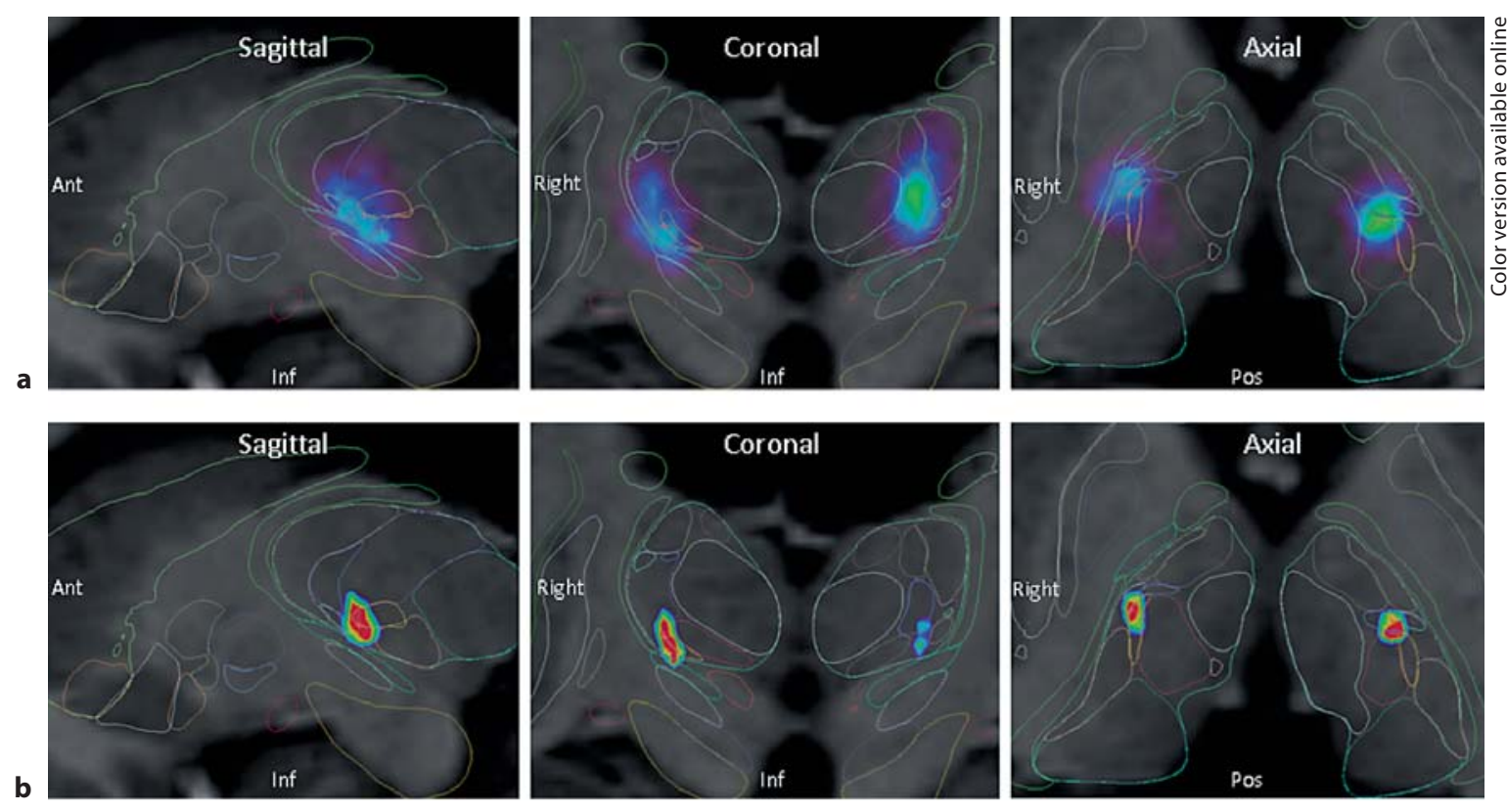

c

0.5

Fig. 1. Sagittal, coronal and axial slices centered on the Vim of the reference MRI volume overlaid with the segmentations of structures and the probabilistic map of tremor control built from a population of patients using Talairach coordinates (a) and nonlinear image normalization (b). c Scale for the probabilistic color maps. For colors, see online version.

listic maps of symptom relief in essential tremor patients. The probabilistic maps are created by using the method described in Pallavaram et al. [24]. Briefly, this consists of centering a sphere with a radius proportional to the level of stimulation that triggered the control of the symptoms at each data point. The probabilistic models are then summed over the whole reference volume and normalized to produce a map ranging between 0 and 1 . It has been extensively reported that the optimal location for symptom relief in essential tremor patients using DBS is in the Vim [25]. Because of the lack of a gold standard, we use an anatomical atlas to show the position of the maps relatively to the anatomy. Not yet as popular as the Schaltenbrand-Wahren atlas [26], the YeB atlas [27] overcomes a number of its limitations by providing the user with an accurate 3-dimensional histological atlas associated with an MRI T1 scan of reference. Using our nonrigid registration algorithm, we projected and overlaid the YeB atlas onto our reference volume. The probabilistic maps were then overlaid on the structures from the YeB atlas. The centroid of the maps as well as their shapes are compared and reported in the next section.

\section{Results}

Figure 1 shows the sagittal, coronal and axial slices centered on the Vim of the reference MRI volume overlaid with the segmentations of structures and the proba- bilistic map of tremor control built from a population of patients using (a) Talairach coordinates and (b) nonlinear image normalization. The scale for the probabilistic maps is shown in figure 1c. We report quantitative results in tables 1 and 2. Table 1 reports the location of the highest probability of tremor control for each normalization method. This high probability region corresponds to the red zone shown in figure $1 \mathrm{~b}$. Table 1 shows that the locations of the centers of the high probability regions in the two maps are quite different. The location based on Talairach normalization is $4.47 \mathrm{~mm}$ posterior, $13.79 \mathrm{~mm}$ lateral and $6.75 \mathrm{~mm}$ superior while that based on nonlinear normalization is $4.47 \mathrm{~mm}$ posterior, $12.12 \mathrm{~mm}$ lateral and $4.38 \mathrm{~mm}$ superior. The Euclidean distance between the two positions is $2.90 \mathrm{~mm}$, which is substantial in the context of the desired precision in DBS surgeries. Table 2 gives information on the shape of the maps in terms of the dimensions of the major axes of the map. This is done by measuring the maximum spread of the probabilistic tremor relief map along the medial-lateral, anterior-posterior and superior-inferior axes. The map based on the Talairach coordinate system has substantial spread compared to the map built using nonlinear registration, 
Table 1. Location of the highest probability of tremor control obtained from the Talairach and nonlinear normalization schemes

\begin{tabular}{lccc}
\hline & A-P & M-L & S-I \\
\hline Talairach coordinate-based normalization & -4.47 & 13.79 & 6.75 \\
Nonlinear normalization & -4.47 & 12.12 & 4.38
\end{tabular}

In millimeters from the mid-commissure. $\mathrm{A}-\mathrm{P}=$ Anteriorposterior; $\mathrm{M}-\mathrm{L}=$ medial-lateral; $\mathrm{S}-\mathrm{I}=$ superior-inferior.

Table 2. Dimensions (in $\mathrm{mm}$ ) of the major and minor axes of the probability map of successful tremor control

A-P $\quad$ M-L S-I
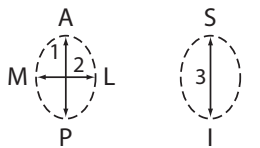

Talairach coordinate-

based normalization

$\begin{array}{lll}6.4 & 3.2 & 7.3\end{array}$

$\begin{array}{llll}\text { Nonlinear normalization } & 2.3 & 1.2 & 1.5\end{array}$

A-P = Anterior-posterior; $\mathrm{M}-\mathrm{L}=$ medial-lateral; $\mathrm{S}-\mathrm{I}=$ superior-inferior.

which is highly localized. The spread of the Talairach system-based map is $6.4 \mathrm{~mm}$ in the anterior-posterior direction, $3.2 \mathrm{~mm}$ in the medial-lateral direction and $7.3 \mathrm{~mm}$ in the superior-inferior direction, while that for the nonlinear registration-based map is only $2.3 \mathrm{~mm}$ in the anterior-posterior direction, $1.2 \mathrm{~mm}$ in the medial-lateral direction and $1.5 \mathrm{~mm}$ in the superior-inferior direction.

\section{Discussion and Conclusion}

Stereotactic and linear methods used to match different brains cannot accurately match functional areas across individuals because these are local subregions that are not well predicted by gross anatomical landmarks. Volumetric approaches such as the nonrigid registration algorithm used in this study can better align such regions allowing more accurate mapping of data from a population of patients and subsequent analyses as illustrated in figure 1 and tables 1 and 2. The results show that statistical maps created by nonlinear normalization of data from several individuals are highly localized while those created using the Talairach coordinates have a larger spread.
This is because nonlinear methods have substantially more degrees of freedom and can thus better account for local anatomical differences. Furthermore, the map built using nonlinear normalization correlates strongly with the anatomical Vim while the Talairach approach produces scattered results.

These findings illustrate the importance of the choice of the normalization scheme while analyzing such data and interpreting the results of such analyses. The use of Talairach coordinates is often favored over other methods because of its simplicity and its prevalence in the medical literature for a long time prior to the availability of more sophisticated normalization schemes. However, even though these new nonlinear methods require additional processing, they should be preferred to linear ones in order to accurately and reliably compare functional information across individuals. Recently, nonlinear algorithms have been made more accessible to researchers and clinicians alike in user-friendly software packages allowing a wider use in clinical analyses. Their use will likely improve the accuracy of statistical studies and clinical conclusions in stereotactic and functional data analyses.

\section{Acknowledgements}

This research has been supported, in parts, by NIH R01 EB006136. The content is solely the responsibility of the authors and does not necessarily represent the official views of these institutes. We thank Medtronic, Inc. and Neurotargeting, LLC. for providing us with the anatomical structures displayed on our atlas from the projection of the YeB atlas.

\section{Disclosure Statement}

Dr. D'Haese and Dr. Dawant are founding members and stock holders and Dr. Pallavaram is a stock holder of Neurotargeting, LLC. that licenses the CRAVE software suite from Vanderbilt University. Dr. Joseph S. Neimat receives financial support from Medtronic Neurological in the form of research grants and consulting fees. Dr. Peter E. Konrad and Dr. Benoit M. Dawant receive financial support from the NIH and Neurotargeting, LLC. in the form of research grants. They are both co-founders of Neurotargeting, LLC., which has licensed some of the technology described in this paper from Vanderbilt University. 


\section{References}

1 Nowinski WL, Belov D, Benabid AL: An algorithm for rapid calculation of a probabilistic functional atlas of subcortical structures from electrophysiological data collected during functional neurosurgery procedures. Neuroimage 2003;18:143-155.

-2 Nowinski WL, Belov D, Thirunavuukarasuu A, Benabid AL: A probabilistic functional atlas of the VIM nucleus constructed from pre-, intra- and postoperative electrophysiological and neuroimaging data acquired during the surgical treatment of Parkinson's disease patients. Stereotact Funct Neurosurg 2005;83:190-196.

3 Guo T, Parrent AG, Peters TM: Automatic target and trajectory identification for deep brain stimulation (DBS) procedures. Med Image Comput Comput Assist Interv 2007; 4791:483-490.

-4 D'Haese P-F, Pallavaram S, Yu H, Spooner J, Konrad PE, Dawant BM: Deformable physiological atlas-based programming of deep brain stimulators: a feasibility study. Lecture Notes Comput Sci Workshop Biomed Image Registration 2006;4057:144-150.

5 Balachandran R, Mitchell J, Dawant BM, Fitzpatrick JM: Accuracy evaluation of MicroTargeting $^{\mathrm{TM}}$ platforms for deep-brain stimulation using virtual targets. IEEE Trans Biomed Eng 2009;56:37-44.

6 D'Haese P-F, Pallavaram S, Konrad PE, Neimat JS, Fitzpatrick JM, Dawant BM: Clinical accuracy of a customized stereotactic platform for deep-brain stimulation after accounting for brain shift. Stereotact Funct Neurosurg 2010;88:81-87.

7 Halpern CH, Danish SF, Baltuch GH, Jaggi JL: Brain shift during deep brain stimulation surgery for Parkinson's disease. Stereotact Funct Neurosurg 2008;86:37-43.

8 Khan MF, Mewes K, Gross RE, Škrinjar O: Assessment of brain shift related to deep brain stimulation surgery. Stereotact Funct Neurosurg 2008;86:44-53.
9 Pallavaram S, Dawant BM, Remple M, Neimat JS, Kao C, Konrad PE, D'Haese PF: Effect of brain shift on the creation of functional atlases for deep brain stimulation surgery. Int J Comput Assist Radiol Surg 2010;5: 221-228.

10 Pallavaram S, D'Haese PF, Remple M, Neimat JS, Kao C, Konrad PE, Dawant BM: A method to correct for brain shift when building electrophysiological atlases for deep brain stimulation (DBS) surgery. Med Image Comput Comput Assist Interv 2009;5761: 557-564.

11 Talairach J, Tournoux P: Co-Planar Stereotaxic Atlas of the Human Brain. Stuttgart, Thieme Publishing Group, 1988.

12 Pallavaram S, Yu H, Spooner J, D'Haese PF, Bodenheimer B, Konrad PE, Dawant BM: Inter-surgeon variability in the selection of anterior and posterior commissures and its potential effects on target localization. Stereotact Funct Neurosurg 2008;86:113-119.

13 Pallavaram S, Dawant BM, Koyama T, Yu H, Neimat JS, Konrad PE, D'Haese PF: Validation of a fully automatic method for the routine selection of the anterior and posterior commissures in MR images. Stereotact Funct Neurosurg 2009;87:148-154.

14 Pluim JP, Maintz JB, Viergever MA: Mutualinformation-based registration of medical images: a survey. IEEE Trans Med Imaging 2003;22:986-1004.

15 Maes F, Collignon A, Suetens P: Multimodality image registration by maximization of mutual information. IEEE Trans Med Imaging 1997;16:187-198.

16 Wells WM, Viola P, Atsumi H, Nakajima S, Kikinis R: Multi-modal volume registration by maximization of mutual information. Med Image Anal 1996;1:35-52.

17 Studholme C, Hill DLG, Hawkes DJ: An overlap invariant entropy measure of $3 \mathrm{D}$ medical image alignment. Pattern Recognit 1999;32:71-86.
18 Chakravarty MM, Sadikot AF, Mongia S, Bertrand G, Collins DL: Towards a multimodal atlas for neurosurgical planning. Med Image Comput Comput Assist Interv 2006; 4191:389-396.

19 Rohde GK, Aldroubi A, Dawant BM: The adaptive bases algorithm for intensity-based nonrigid image registration. IEEE Trans Med Imaging 2003;22:1470-1479.

20 Rohr K, Stiehl HS, Buzug TM, Weese J, Kuhn $\mathrm{MH}$ : Landmark-based elastic registration using approximating thin-plate splines. IEEE Trans Med Imaging 2001;20:526-534.

21 Wenhong S, Weidong Z, Mingqiang Y: Medical image registration using thin-plate spline for automatically detecting and matching of point sets. 5th Int Conf Bioinformatics Biomed Eng, Wuhan, 2011, pp 1-4.

22 Zhiyu Q, Huihui T, Dongsheng T: Non-rigid medical image registration based on the thin-plate spline algorithm. WRI World Congr Comput Sci Information Eng, Los Angeles, 2009, pp 522-527.

23 Bookstein FL: Principal warps: thin-plate splines and the decomposition of deformations. IEEE Trans Pattern Anal Mach Intell 1989;11:567-585.

24 Pallavaram S, D'Haese PF, Kao C, Yu H, Remple M, Neimat JS, Konrad PE, Dawant BM: A new method for creating electrophysiological maps for DBS surgery and their application to surgical guidance. Med Image Comput Comput Assist Interv 2008;5241: 670-677.

25 Lozano AM: Vim thalamic stimulation for tremor. Arch Med Res 2000;31:266-269.

26 Schaltenbrand G, Wahren W: Atlas for Stereotaxy of the Human Brain. Stuttgart, Thieme, 1977.

27 Yelnik J, Bardinet E, Dormont D, Malandain G, Ourselin S, Tandé D, Karachi C, Ayache N, Cornu P, Agid Y: A three-dimensional, histological and deformable atlas of the human basal ganglia. 1. Atlas construction based on immunohistochemical and MRI data. Neuroimage 2007;34:618-638. 